Network Working Group

Request for Comments: 4510

Obsoletes: 2251, 2252, 2253, 2254, 2255,

$2256,2829,2830,3377,3771$

Category: Standards Track
K. Zeilenga, Ed. OpenLDAP Foundation June 2006

\title{
Lightweight Directory Access Protocol (LDAP) : Technical Specification Road Map
}

Status of This Memo

This document specifies an Internet standards track protocol for the Internet community, and requests discussion and suggestions for improvements. Please refer to the current edition of the "Internet Official Protocol Standards" (STD 1) for the standardization state and status of this protocol. Distribution of this memo is unlimited.

Copyright Notice

Copyright (C) The Internet Society (2006).

Abstract

The Lightweight Directory Access Protocol (LDAP) is an Internet protocol for accessing distributed directory services that act in accordance with $\mathrm{X} .500$ data and service models. This document provides a road map of the LDAP Technical Specification.

1. The LDAP Technical Specification

The technical specification detailing version 3 of the Lightweight Directory Access Protocol (LDAP), an Internet Protocol, consists of this document and the following documents:

LDAP: The Protocol [RFC4511]

LDAP: Directory Information Models [RFC4512]

LDAP: Authentication Methods and Security Mechanisms [RFC4513]

LDAP: String Representation of Distinguished Names [RFC4514]

LDAP: String Representation of Search Filters [RFC4515]

LDAP: Uniform Resource Locator [RFC4516]

LDAP: Syntaxes and Matching Rules [RFC4517]

LDAP: Internationalized String Preparation [RFC4518]

LDAP: Schema for User Applications [RFC4519] 
The terms "LDAP" and "LDAPv3" are commonly used to refer informally to the protocol specified by this technical specification. The LDAP suite, as defined here, should be formally identified in other documents by a normative reference to this document.

LDAP is an extensible protocol. Extensions to LDAP may be specified in other documents. Nomenclature denoting such combinations of LDAP-plus-extensions is not defined by this document but may be defined in some future document(s). Extensions are expected to be truly optional. Considerations for the LDAP extensions described in BCP 118, RFC 4521 [RFC4521] fully apply to this revision of the LDAP Technical Specification.

IANA (Internet Assigned Numbers Authority) considerations for LDAP described in BCP 64, RFC 4520 [RFC4520] apply fully to this revision of the LDAP technical specification.

\subsection{Conventions}

The key words "MUST", "MUST NOT", "REQUIRED", "SHALL", "SHALL NOT", "SHOULD", "SHOULD NOT", "RECOMMENDED", "MAY", and "OPTIONAL" in this document are to be interpreted as described in BCP 14 [RFC2119].

2. Relationship to X.500

This technical specification defines LDAP in terms of [X.500] as an X.500 access mechanism. An LDAP server MUST act in accordance with the X.500 (1993) series of International Telecommunication Union Telecommunication Standardization (ITU-T) Recommendations when providing the service. However, it is not required that an LDAP server make use of any X.500 protocols in providing this service. For example, LDAP can be mapped onto any other directory system so long as the X.500 data and service models [X.501][X.511], as used in LDAP, are not violated in the LDAP interface.

This technical specification explicitly incorporates portions of X.500(93). Later revisions of X.500 do not automatically apply to this technical specification.

3. Relationship to Obsolete Specifications

This technical specification, as defined in Section 1, obsoletes entirely the previously defined LDAP technical specification defined in RFC 3377 (and consisting of RFCs 2251-2256, 2829, 2830, 3771, and 3377 itself). The technical specification was significantly reorganized. 
This document replaces RFC 3377 as well as section 3.3 of RFC 2251. [RFC4512] replaces portions of RFC 2251, RFC 2252, and RFC 2256. [RFC4511] replaces the majority RFC 2251, portions of RFC 2252, and all of RFC 3771. [RFC4513] replaces RFC 2829, RFC 2830, and portions of RFC 2251. [RFC4517] replaces the majority of RFC 2252 and portions of RFC 2256. [RFC4519] replaces the majority of RFC 2256. [RFC4514] replaces RFC 2253. [RFC4515] replaces RFC 2254. [RFC4516] replaces RFC 2255.

[RFC4518] is new to this revision of the LDAP technical specification.

Each document of this specification contains appendices summarizing changes to all sections of the specifications they replace. Appendix A.1 of this document details changes made to RFC 3377. Appendix A.2 of this document details changes made to section 3.3 of RFC 2251 .

Additionally, portions of this technical specification update and/or replace a number of other documents not listed above. These relationships are discussed in the documents detailing these portions of this technical specification.

4. Security Considerations

LDAP security considerations are discussed in each document comprising the technical specification.

5. Acknowledgements

This document is based largely on RFC 3377 by J. Hodges and R. Morgan, a product of the LDAPBIS and LDAPEXT Working Groups. The document also borrows from RFC 2251 by M. Wahl, T. Howes, and S. Kille, a product of the ASID Working Group.

This document is a product of the IETF LDAPBIS Working Group. 
6. Normative References

[RFC2119] Bradner, S., "Key words for use in RFCs to Indicate Requirement Levels", BCP 14, RFC 2119, March 1997.

[RFC4511] Sermersheim, J., Ed., "Lightweight Directory Access Protocol (LDAP): The Protocol", RFC 4511, June 2006.

[RFC4512] Zeilenga, K., "Lightweight Directory Access Protocol (LDAP): Directory Information Models", RFC 4512, June 2006 .

[RFC4513] Harrison, R., Ed., "Lightweight Directory Access Protocol (LDAP): Authentication Methods and Security Mechanisms", RFC 4513, June 2006.

[RFC4514] Zeilenga, K., Ed., "Lightweight Directory Access Protocol (LDAP): String Representation of Distinguished Names", RFC 4514, June 2006.

[RFC4515] Smith, M., Ed. and T. Howes, "Lightweight Directory Access Protocol (LDAP): String Representation of Search Filters", RFC 4515, June 2006.

[RFC4516] Smith, M., Ed. and T. Howes, "Lightweight Directory Access Protocol (LDAP): Uniform Resource Locator", RFC 4516, June 2006 .

[RFC4517] Legg, S., Ed., "Lightweight Directory Access Protocol (LDAP): Syntaxes and Matching Rules", RFC 4517, June 2006 .

[RFC4518] Zeilenga, K., "Lightweight Directory Access Protocol (LDAP) : Internationalized String Preparation", RFC 4518, June 2006 .

[RFC4519] Sciberras, A., Ed., "Lightweight Directory Access Protocol (LDAP): Schema for User Applications", RFC 4519, June 2006 .

[RFC4520] Zeilenga, K., "Internet Assigned Numbers Authority (IANA) Considerations for the Lightweight Directory Access Protocol (LDAP)", BCP 64, RFC 4520, June 2006.

[RFC4521] Zeilenga, K., "Considerations for LDAP Extensions", BCP 118, RFC 4521, June 2006. 
$[\mathrm{X} .500]$

International Telecommunication Union Telecommunication Standardization Sector, "The Directory -- Overview of concepts, models and services", X.500(1993) (also ISO/IEC 9594-1:1994).

[X.501] International Telecommunication Union Telecommunication Standardization Sector, "The Directory -- Models", X.501(1993) (also ISO/IEC 9594$2: 1994)$.

[X.511] International Telecommunication Union Telecommunication Standardization Sector, "The Directory: Abstract Service Definition", X.511(1993) (also ISO/IEC 9594-3:1993). 
Appendix A. Changes to Previous Documents

This appendix outlines changes this document makes relative to the documents it replaces (in whole or in part).

\section{A.1. Changes to RFC 3377}

This document is nearly a complete rewrite of RFC 3377 as much of the material of RFC 3377 is no longer applicable. The changes include redefining the terms "LDAP" and "LDAPv3" to refer to this revision of the technical specification.

A.2. Changes to Section 3.3 of RFC 2251

The section was modified slightly (the word "document" was replaced with "technical specification") to clarify that it applies to the entire LDAP technical specification.

Author's Address

Kurt D. Zeilenga

OpenLDAP Foundation

EMail: Kurt@OpenLDAP.org 
Full Copyright statement

Copyright (C) The Internet Society (2006).

This document is subject to the rights, licenses and restrictions contained in BCP 78, and except as set forth therein, the authors retain all their rights.

This document and the information contained herein are provided on an "AS IS" basis and THE CONTRIBUTOR, THE ORGANIZATION HE/SHE REPRESENTS OR IS SPONSORED BY (IF ANY), THE INTERNET SOCIETY AND THE INTERNET ENGINEERING TASK FORCE DISCLAIM ALL WARRANTIES, EXPRESS OR IMPLIED, INCLUDING BUT NOT LIMITED TO ANY WARRANTY THAT THE USE OF THE INFORMATION HEREIN WILL NOT INERINGE ANY RIGHTS OR ANY IMPLIED WARRANTIES OF MERCHANTABILITY OR FITNESS FOR A PARTICULAR PURPOSE.

Intellectual Property

The IETF takes no position regarding the validity or scope of any Intellectual Property Rights or other rights that might be claimed to pertain to the implementation or use of the technology described in this document or the extent to which any license under such rights might or might not be available; nor does it represent that it has made any independent effort to identify any such rights. Information on the procedures with respect to rights in RFC documents can be found in BCP 78 and BCP 79 .

Copies of IPR disclosures made to the IETF Secretariat and any assurances of licenses to be made available, or the result of an attempt made to obtain a general license or permission for the use of such proprietary rights by implementers or users of this specification can be obtained from the IETF on-line IPR repository at http://www.ietf.org/ipr.

The IETF invites any interested party to bring to its attention any copyrights, patents or patent applications, or other proprietary rights that may cover technology that may be required to implement this standard. Please address the information to the IETF at ietf-ipreietf.org.

Acknowledgement

Funding for the RFC Editor function is provided by the IETF Administrative Support Activity (IASA) . 